\title{
From Arctic to Antarctic: Is There any Remaining Role for Platelet Function Testing to Tailor Therapy after Coronary Stent Implantation? Cheuk-Kit Wong*
}

Department of Medicine and Therapeutics, Faculty of Medicine, The Chinese University of Hong Kong, Hong Kong

The recently reported ANTARCTIC trial [1] is an open-label, blinded endpoint-adjudication, randomised controlled superiority study from 35 centres in France testing the role of tailored antiplatelet therapy among 877 elderly patients ( $41 \%$ women and $28 \%$ diabetics) over the age of 75 years ( 20\% over 85 ) with either ST elevation or non-ST elevation acute myocardial infarction undergoing coronary stenting.

Patients (recruited between March 2012 and May 2015) were started on prasugrel $5 \mathrm{mg}$ daily and randomized to tailored antiplatelet therapy $(n=435)$ versus conventional therapy $(n=442)$. Platelet function was assessed by the point-of-care VerifyNow P2Y $\mathrm{Y}_{12}$ cartridge platelet function test performed on days 14 and 28 . If platelet reactivity unit (PRU) was $\geq 208$, prasugrel was increased to $10 \mathrm{mg}$ daily; and if $\leq 85$, prasugrel was changed to clopidogrel $75 \mathrm{mg}$ daily.

The trial excluded patients with previous history of transient ischaemic attack or stroke, fibrinolytic therapy within the past $48 \mathrm{~h}$, chronic oral anticoagulation, concomitant medical illness with reduced survival, allergy or intolerance to aspirin or thienopyridines, active bleeding or history of bleeding diathesis, thrombocytopenia, severe hepatic impairment, or conditions potentially associated with poor treatment adherence such as dementia.

It turned out that in the tailored antiplatelet therapy group only $3.7 \%$ required escalation to prasugrel $10 \mathrm{mg}$ daily while $39.3 \%$ were converted to clopidogrel $75 \mathrm{mg}$ daily. The primary outcome, measuring the composite of cardiovascular death, myocardial infarction, stroke, stent thrombosis, urgent revascularization, or bleeding complication at 1 year, occurred in $27.6 \%$ of the tailored therapy group vs. $27.8 \%$ of the conventional therapy group $(\mathrm{p}=0.98)$. Secondary outcome measuring the composite of cardiovascular death, myocardial infarction, stent thrombosis, or urgent revascularization was also not different, $9.9 \%$ vs. $9.3 \%$ respectively $(\mathrm{p}=0.80)$.

The ANTARCTIC authors concluded that platelet function monitoring with treatment adjustment did not improve clinical outcomes. They further remarked in their concluding paragraph of the abstract that ".....platelet function testing is being used in many centres and international guidelines still recommend platelet function testing in high-risk situations. Our study does not support this practice or these recommendations."

\section{Where Do We Stand with Anti-Platelet Therapy Post- Coronary Stenting?}

The basis for dual antiplatelet agents (aspirin plus a $\mathrm{P}_{2} \mathrm{Y}_{12}$ receptor antagonist) in preventing acute and sub-acute thrombosis after coronary stenting was established 2 decades ago. The era of drug eluting stents from the last decade onwards ushered the practice of prolonged dual antiplatelet therapy in the hope of preventing late stent thrombosis. With this approach comes the conundrum between antithrombotic benefit versus bleeding risk, hence the quest for a potential "sweet spot" of platelet suppression akin to the "therapeutic INR range" with warfarin therapy. As such it will be appropriate to review how (and why) ANTARCTIC was designed before exploring why the superiority trial turned out to be negative.
Few years ago the French ARCTIC trial was reported [2], randomizing 2440 patients scheduled for coronary stenting to a strategy of "tailored anti-platelet therapy" or conventional therapy. Tailored therapy was by point-of-care VerifyNow P2 $\mathrm{Y}_{12}$ cartridge and aspirin cartridge assays. These assays were performed right before stent implantation and in the outpatient clinic 2-4 weeks later. For patients having high platelet reactivity despite clopidogrel (34.5\% of patients) or aspirin (7.6\%), they were given additional bolus of clopidogrel, prasugrel, or aspirin along with intra-procedural glycoprotein IIb/ IIIa inhibitors. ARCTIC excluded patients with ST elevation acute myocardial infarction.

The primary end point in ARCTIC measured the composite of death, myocardial infarction, stent thrombosis, stroke, or urgent revascularization 1 year after stenting. This occurred in $34.6 \%$ of the patients in the tailored therapy group vs. $31.1 \%$ in the conventional treatment group $(p=0.10)$. The main secondary end point (stent thrombosis or any urgent revascularization) occurred in $4.9 \%$ in the tailored therapy group vs. $4.6 \%$ in the conventional treatment group $(\mathrm{P}=0.77)$. Stent thrombosis rate was $1.0 \%$ vs. $0.7 \%$ respectively $(\mathrm{p}=0.51)$. The rate of major bleeding events also did not differ significantly.

An editorial in this journal commenting on ARCTIC questioned whether measuring platelet reactivity after clopidogrel could be reaching the end of the road [3], given that more efficacious antiplatelet medications such as prasugrel were becoming available. Indeed in ANTARCTIC, a "low" dose of prasugrel of $5 \mathrm{mg}$ turned out to give sufficiently adequate platelet suppression in $>96 \%$ of patients.

The ANTARCTIC investigators [1] explained that given the failure of the tailored antiplatelet therapy approach in ARCTIC (and in other trials with similar design but focussing on lower-risk patients) there was a need to investigate real high-risk patients than those undergoing elective coronary stenting for stable or stabilized coronary disease. Thus ANTARCTIC enrolled only elderly patients with acute myocardial infarction, who were at increased risk for both ischaemic events and bleeding. Indeed the events observed was higher than the event rates anticipated in their sample-size calculation, with $27.7 \%$ events for the primary endpoint versus an expected rate of $19.5 \%$.

Despite the high event rates which increased statistical power, ANTARCTIC showed that tailored antiplatelet therapy did not

*Corresponding author: Cheuk-Kit Wong, Adjunct Professor, Department of Medicine and Therapeutics, Faculty of Medicine, The Chinese University of Hong Kong, Prince of Wales Hospital, Shatin, Hong Kong, Tel: 852-26323139; E-mail: cheuk-kit.wong@cuhk.edu.hk

Received September 25, 2016; Accepted September 27, 2016; Published October 03, 2016

Citation: Wong CK (2016) From Arctic to Antarctic: Is There any Remaining Role for Platelet Function Testing to Tailor Therapy after Coronary Stent Implantation? Cardiovasc Pharm Open Access 5: e132. doi: 10.4172/2329-6607.1000e132

Copyright: (C) 2016 Wong CK. This is an open-access article distributed under the terms of the Creative Commons Attribution License, which permits unrestricted use, distribution, and reproduction in any medium, provided the original author and source are credited. 
improve outcomes. Although $\sim 40 \%$ of patients were down-titrated to clopidogrel bleeding events were not reduced.

\section{Why ANTARCTIC Failed and What Remains for Tailored Antiplatelet Therapy After Stenting with Prasugrel?}

More modern stent designs such as second generation drug eluting stents (compared to their first generation counterparts) have reduced the risks of stent thrombosis so it is important to interpret results in the context of stent design evolution for which the "year of patient recruitment" acts as a surrogate.

In the ADAPT-DES 8665 patient registry [4], a prospective, multicentre registry of patients with one or more drug eluting stents implantation and receiving aspirin and clopidogrel (patients recruited between Jan 2008 and Sept, 2010), the rate of stent thrombosis over 1 year was only $0.8 \%$ with most cases occurring within the first 2 weeks of stenting. High platelet reactivity on clopidogrel, as observed in $\sim 40 \%$ of patients early after stenting, was related to subsequent stent thrombosis (adjusted hazard ratio 2.49 [95\% CI 1.43-4.31]) and myocardial infarction (adjusted hazard ratio 1.42 [95\% CI 1.09-1.86]), but was inversely related to bleeding (adjusted hazard ratio $0.73[95 \%$ CI 0.61-0.89]) [4].

The ANTARCTIC trial design with tailored antiplatelet (thienopyridine) therapy after the first 2 weeks was not so much testing the treatment efficacy in preventing subsequent stent thrombosis (because of the expected ultra-low incidence) but rather testing the general effects of this tailored platelet inhibition in the elderly patients stented for an acute myocardial infarction.

One distinct area of missing information in the ANTARCTIC publication [1] is events related to occurrences of atrial fibrillation. Atrial fibrillation could have been quite common in ANTARCTIC (elderly post-myocardial infarction) patients. The potentially differential use of anti-coagulants among the 2 randomized groups (given the open-label ANTARCTIC trial design) might have confounded their results.

The thienopyridines prasugrel and clopidogrel are pro-drugs. After intestinal absorption, clopidogrel requires two cytochrome P-450 (CYP)-dependent oxidation steps to generate its active metabolite. For prasugrel, it is rapidly hydrolysed in the intestinal wall to an intermediate compound and requires a further CYP-dependent oxidation step to generate its active metabolite. Relevant CYP isoenzymes involved in activating clopidogrel (and to a lesser extent prasugrel) may be affected by genetic polymorphisms which lead to different levels of their final active metabolites binding irreversibly to the platelet $\mathrm{P} 2 \mathrm{Y}_{12}$ receptors. For clopidogrel, patients with either 1 or 2 loss-of-function CYP2C19 alleles are known to have an attenuated pharmacologic response and worse clinical outcomes, with greater impairment in those who had both alleles affected [3].

Given that most patients already responded to the "low" dose prasugrel of $5 \mathrm{mg}$ daily in ANTARCTIC [1], one explanation for the negative result is that there is actually no precise "sweet spot" PRU once on prasugrel: ie. more intensive platelet suppression with lower PRU will reduce ischemic event but increase bleeding events, and vice-versa. The situation is different with the use of clopidogrel for which up to $40 \%$ of patients are somewhat resistant [4] with the occasional patients (often having 2 loss-of-function CYP2C19 alleles) actually having minimal platelet suppression and potentially unduly high risks of stent thrombosis. The latter situation should be remedied by switching clopidogrel to prasugrel or ticagrelor [3].
On the other hand, the ANTARCTIC data suggest that the option of stepping-down therapy according to platelet function test 2 weeks after stenting (as happened in $\sim 40 \%$ of patients) is potentially viable. As shown in their supplementary data [1], platelet function was reassessed on Day 28 in 148 of the 182 patients who were converted to clopidogrel from Day 14. Of these 148 patients, 55 continued to have low $\mathrm{PRU}<85$, 6 had high PRU $>208$ and 87 had an optimal PRU. Subset data on the outcome of this "step-down" group was not reported [1], but is not expected to differ much from the whole tailored therapy group as only $3.7 \%$ of patients in the tailored therapy group had "step-up" prasugrel therapy. If this is confirmed, the tailored "step-down" approach will at least be cost-saving.

\section{Observational Studies and Randomized Trials-What Questions Remain after ANTARCTIC?}

The cut-points for determining high PRU were obtained from observational studies [3]. The ADAPT-DES registry supported the use of the PRU cut-point of 208 on the VerifyNow P2Y ${ }_{12}$ cartridge platelet function test [4]. Also in ADAPT-DES, high platelet reactivity on aspirin was not significantly associated with stent thrombosis (adjusted hazard ratio 1.46 [0.58-3.64]), myocardial infarction, or death, but was inversely related to bleeding (adjusted hazard ratio 0.65 [0.43-0.99]) [4]. However, observational data are confounded by measured and unmeasured parameters and open to different ways of analysis.

An editorial in the journal last year [5] discussed the very different conclusions reached by 2 prominent academic groups analysing data on door-to-balloon time in the US Cath-PCI registry. The first group showed unchanged in-hospital mortality from 2005 through 2009 for STEMI despite significant shortened door-to-balloon time [6], while re-analysis of the same data (with minor extensions) by another group showed positive and almost linear relationship between door-toballoon time and in-hospital mortality within every calendar year [7].

Randomized controlled trial is the gold standard of evidence-based medicine. The findings from ANTARCTIC thus deserve due respect. ANTARCTIC is unique as a dedicated trial for elderly subjects post myocardial infarction, and the authors are correct to conclude that their findings do not support a role of platelet function testing 2 weeks post-stenting to tailor thienopyridine therapy. At this time-point the majority of stent thrombosis cases would have occurred, but there remain other high risk situations mainly pertaining to bleeding. An example will be patients who require concomitant anti-coagulants (an exclusion criterion in ANTARCTIC) who may benefit from tailored therapy according to platelet function testing. Future studies should address these problems.

\section{References}

1. Cayla G, Cuisset T, Silvain J, Leclercq F, Manzo-Silberman S, et al. (2016) Platelet function monitoring to adjust antiplatelet therapy in elderly patients stented for an acute coronary syndrome (ANTARCTIC): an open-label, blindedendpoint, randomised controlled superiority trial. Lancet.

2. Collet JP, Cuisset T, Rangé G, Cayla G, Elhadad S, et al. (2012) Bedside monitoring to adjust antiplatelet therapy for coronary stenting. $\mathrm{N}$ Engl J Med 367: 2100-2109.

3. Wong CK (2012) Measuring Platelet Reactivity after Clopidogrel-Has it Reached the End of the Road? Cardiovasc Pharm Open Access 1: e105.

4. Stone GW, Witzenbichler B, Weisz G, Rinaldi MJ, Neumann FJ, et al. (2013) Platelet reactivity and clinical outcomes after coronary artery implantation of drug-eluting stents (ADAPT-DES): a prospective multicentre registry study. Lancet 382: 614-623.

5. Wong CK (2015) Time for a reappraisal: How much has the last 10 years of "mainstream" STEMI research impacted on STEMI outcome? Cardiovasc Pharm Open Access 4: e129. 
Citation: Wong CK (2016) From Arctic to Antarctic: Is There any Remaining Role for Platelet Function Testing to Tailor Therapy after Coronary Stent Implantation? Cardiovasc Pharm Open Access 5: e132. doi: 10.4172/2329-6607.1000e132

6. Menees DS, Peterson ED, Wang Y, Curtis JP, Messenger JC, et al. (2013) Door-to-balloon time and mortality among patients undergoing primary $\mathrm{PCl}$. N Engl J Med 369: 901-909.
7. Nallamothu BK, Normand SL, Wang Y, Hofer TP, Brush JE, et al. (2015) Relation between door-to-balloon times and mortality after primary percutaneous coronary intervention over time: a retrospective study. Lancet 385: 1114-1122. 